\title{
Agénésie bilatérale des vésicules séminales et des canaux déférents
}

\author{
Amadou Kassogué, MD; ${ }^{*}$ J. Eddine El Ammari, MD; ${ }^{*}$ Alkadri Diarra, MD; ${ }^{*}$ Driss Amiroune, MD;* \\ Mustapha Ahsaini, MD; Karim Ouldim, MD; Zacharia Traoré, MD; ${ }_{;}^{*}$ H. Nadia Sqalli, MD,; Siham Tizniti, MD; \\ Soufiane Mellas, MD; ${ }^{*}$. Fadl Tazi, MD; ${ }^{*}$ Abdelhak Khallouk, MD; ${ }^{*}$. Jamal El Fassi, MD;* My Hassan Farih, MD*
}

*Service d'urologie, CHU Hassan II-Fès, Maroc; †Service d'anatomie pathologie et de cytologie, Unité de cytogénétique et biologie moléculaire, CHU Hassan II-Fès, Maroc; §Service d'imagerie médicale, CHU Hassan II-Fès, Maroc

Cite as: Can Urol Assoc J 2014;8(7-8):e490-2. http://dx.doi.org/10.5489/cuaj.1503

Published online July 18, 2014.

\section{Résumé}

L'absence de canaux déférents et de vésicules séminales est une cause très rare d'infertilité masculine qui pose des difficultés d'ordre diagnostique. De plus, son traitement est complexe. Dans le présent article, nous rapportons le cas d'un patient âgé de 43 ans qui a consulté pour infertilité primaire évoluant depuis 5 ans (un conseil génétique a été réalisé) et nous examinons les difficultés diagnostiques et thérapeutiques liées à cette forme particulière d'infertilité.

\section{Introduction}

L'absence de canaux déférents et de vésicules séminales est une cause très rare d'infertilité masculine. L'agénésie bilatérale des canaux déférents $(A B C D)$ est la plus fréquente des anomalies des voies génitales masculines découvertes à l'âge adulte au cours d'un bilan d'azoospermie excrétoire' L'infertilité liée à des anomalies des organes génitaux internes est rare ; elle est estimée à $2 \%$ des cas de stérilité masculine $^{2}$. Nous rapportons le cas d'un patient et examinons les difficultés d'ordre diagnostique et thérapeutique liées à cette forme particulière d'infertilité.

\section{Observation}

Un patient de 43 ans, marié depuis 5 ans, a été admis en consultation pour infertilité du couple. L'examen des organes génitaux externes (verge, scrotum, orifices herniaires, testicules) n'a permis de déceler aucune anomalie. La palpation du trajet du cordon spermatique a été effectuée afin de vérifier la présence des canaux déférents et a soulevé un doute quant à leur étroitesse ou à leur absence. Un sper- mogramme réalisé à deux reprises a indiqué une azoospermie. Le bilan hormonal (FSH, LH, testostéronémie) était normal. Une échographie endorectale a mis en lumière l'absence des vésicules séminales. Une imagerie par résonance magnétique (IRM) pelvienne a confirmé l'agénésie bilatérale des vésicules séminales et des canaux déférents (Fig. 1). L'analyse cytogénétique (caryotype sanguin) a permis de conclure à l'absence d'anomalies chromosomiques sur toutes les mitoses observées dans la limite de la résolution du caryotype métaphasique. Le patient a été adressé à un centre de procréation assistée pour une injection intracytoplasmique de spermatozoïde.

\section{Discussion}

L'absence de canaux déférents $(A C D)$ et de vésicules séminales est une cause très rare d'infertilité masculine. L'ABCD est la plus fréquente des anomalies des voies génitales masculines découvertes à l'âge adulte au cours d'un bilan d'azoospermie excrétoire ${ }^{1}$. L'ACD est une entité particulière parmi les causes de stérilité masculine ; elle serait responsable de seulement $2 \%$ des cas. En dehors de la mucoviscidose, qui sous-tend généralement une absence des canaux déférents, cette entité a été longtemps considérée comme une forme rare et isolée de déterminisme génétique autosomique récessif. Cependant, avec l'identification, en 1989, du gène impliqué dans la mucoviscidose, on a démontré, dès 1990, une parenté génétique entre l'absence isolée des canaux déférents et les mutations du gène de la mucoviscidose ${ }^{3}$.

Les examens cliniques, échographiques et génétiques permettent d'envisager différentes possibilités : absence unilatérale ou bilatérale des canaux déférents, isolée ou associée à une agénésie rénale, avec absence ou non des vésicules séminales, avec ou sans mutation de la mucoviscidose. L'échographie endorectale est un examen fiable qui permet de confirmer le diagnostic d'absence des vésicules séminales suspectée en cours d'examen clinique. Les patients qui 
présentent une $\mathrm{ACD}$ isolée, associée à une mutation de la mucoviscidose, courent un risque de transmettre la maladie. Ce risque est estimé a priori à 1/50. Dans les cas d'ACD isolée et associée à une mutation de la mucoviscidose, la recherche d'une mutation génétique chez la conjointe et un conseil génétique sont nécessaires avant d'entreprendre la procréation médicalement assistée ${ }^{3-4}$. Dans le cas qui nous occupe, I'ACD était bilatérale et associée à une agénésie des vésicules séminales. La recherche de la mutation génétique chez le patient a donné des résultats négatifs ; il n'y avait pas de gène de mutation de la mucoviscidose. La recherche d'une mutation génétique chez la conjointe n'a pas été nécessaire avant d'entreprendre la procréation médicalement assistée.

Selon l'étude de Robin G et coll. ${ }^{5}$, une échographie des voies génitales profondes par voie transrectale, réalisée à titre systématique dans I'exploration de cette infertilité et complétée $d^{\prime}$ une IRM pelvienne, a montré une agénésie unilatérale isolée du canal déférent gauche dans la portion pelvienne. Étant donné la présence de cette anomalie, la recherche de mutations du gène de CFTR (cystic fibrosis transmembrane conductance regulator, ou régulateur de la perméabilité transmembranaire de la fibrose kystique) a mis en évidence une hétérozygotie composite (Delta F508/ V938G). C'est cette étude qui a fait mention, pour la pre-

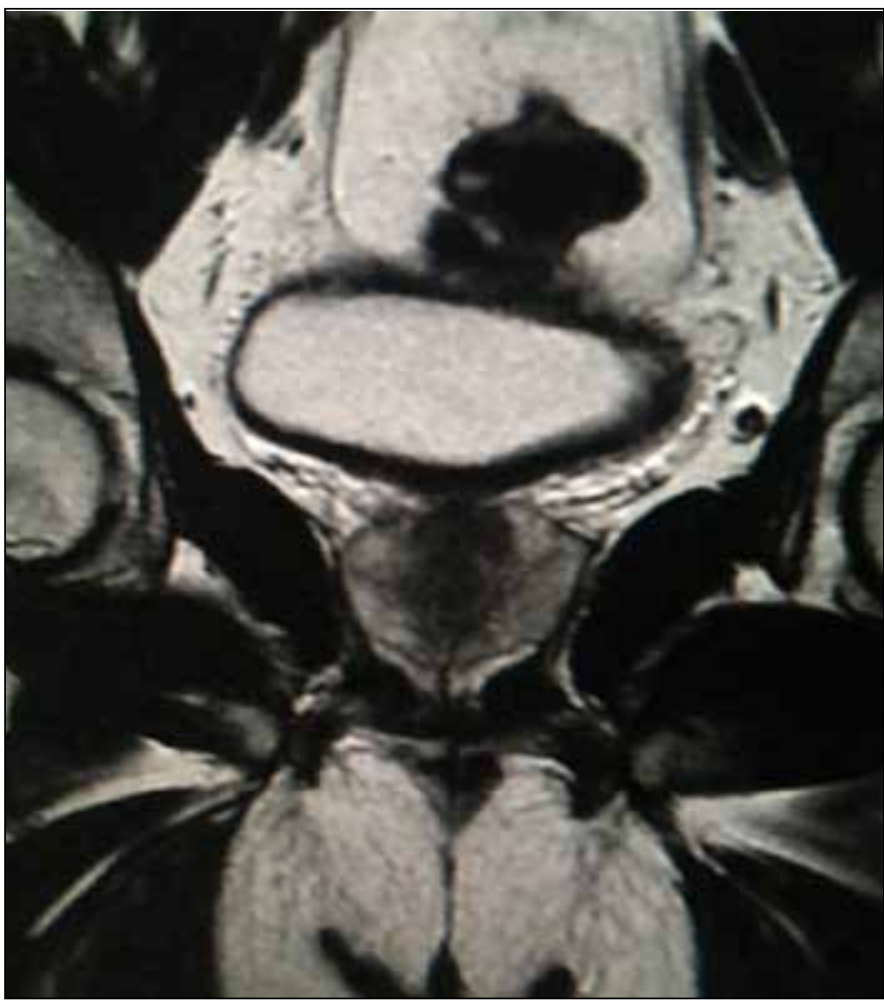

Fig. 1. Une imagerie par résonance magnétique des voies séminales profondes en coupe coronale en séquence T2. Mise en évidence de la prostate. Absence de visualisation des vésicules séminales et des canaux déférents. mière fois, de l'association de ces deux mutations dans la littérature ${ }^{5}$. Chez notre patient, le diagnostic d'absence de vésicules séminales a été posé à l'échographie endorectale et confirmé par I'IRM pelvienne. Ces deux examens permettent de confirmer le diagnostic d'agénésie des canaux déférents et des vésicules séminales.

Les malformations des organes génitaux internes masculins issus du canal mésonéphrotique, ou canal de Wolff (épididyme, canaux déférents, vésicules séminales, canal éjaculateur), sont des pathologies mal connues en raison de leur rareté. L'agénésie déférentielle est le plus souvent bilatérale. Elle se caractérise par l'absence des vésicules séminales et du canal éjaculateur. La mutation du gène CFTR contribue aux anomalies du développement des canaux déférents ${ }^{6,7}$. En présence d'agénésie déférentielle, il faut rechercher une mucoviscidose. Chez l'adulte, le point d'appel principal des agénésies bilatérales est l'anomalie du spermogramme ${ }^{6}$.

L'absence congénitale bilatérale des canaux déférents et l'absence congénitale unilatérale des canaux déférents sont deux causes d'infertilité masculine. On les retrouve chez de $1 \%$ à $2 \%$ des hommes examinés pour infertilité et environ $10 \%$ des hommes atteints d'azoospermie ${ }^{8}$.

\section{Conclusion}

L'absence de canaux déférents et de vésicules séminales est une cause très rare d'infertilité masculine. L'échographie endorectale et I'IRM pelvienne sont deux examens fiables qui permettent de confirmer le diagnostic d'absence des canaux déférents et des vésicules séminales. Le conseil génétique est indispensable pour déceler une maladie génétique associée. La procréation médicalement assistée par injection intracytoplasmique de spermatozoïde permet de résoudre le problème d'infertilité du couple.

This paper has been peer-reviewed.

Competing interests: Dr. Kassogué, Dr. El Ammari, Dr. Diarra, Dr. Amiroune, Dr. Ahsaini, Dr. Ouldim, Dr. Traoré, Dr. Sqalli, Dr. Tizniti, Dr. Mellas, Dr. Tazi, Dr. Khallouk, Dr. El Fassi and Dr. Farih all declare no competing financial or personal interests.

\section{Références}

1. Merrot T, Delarue A, Chaumoitre $\mathrm{K}$, et al. Agénésie déférentielle bilatérale et hernie inguinale chez I'enfant. Une présentation précoce rare de la mucoviscidose. Arch Pediatr 2001;8:728-30. http:// dx.doi.org/10.1016/S0929-693X(00)90306-3

2. Fontaine $E$, Jardin $A$. Anomalies des organes génitaux internes masculins et retentissement sur la fertilité. Prog Urol 2001;11:729-32.

3. Robert F, Rollet J, Lapray JF, et al. Les absences des canaux déférents dans l'infertilité masculine. Tentative de classification à partir de 39 cas. Presse Med 1999;28:116-2.

4. Desideri Vaillant C, Creff J, et al. Stratégies d'exploration fonctionnelle et de suivi thérapeutique. Implication du gène CFTR dans la stérilité masculine associée à une absence des canaux déférents. Immuno-analyse et Biologie spécialisée 2004;19:343-50. http://dx.doi.org/10.1016/i.immbio.2004.09.003 
Kassogué et al.

5. Robin G, Lefebvre Khali V, Dumur V, et al. Agénésie déférentielle partielle unilatérale et hétérozygotie composite du gène CFTR (Delta F508/V938G). Gynecol Obstet Fertil 2007;35:561-4. http://dx.doi. org/10.1016/i.gyobfe.2006.12.026

6. Dubois $R$, Valvalle AF, Murat $F$, et al. Malformations des organes génitaux internes masculins issus du canal mésonéphrotique de Wolff. Prog Urol 2001;11:733-40.

7. Samli H, Samli MM, Yilmaz E, et al. Clinical, andrological and genetic characteristics of patients with congenital bilateral absence of vas deferens (CBAVD). Arch Androl 2006;52:471-7. http://dx.doi. org/10.1080/01485010600691993
8. Radpour R, Gourabi H, Gilani MA, et al. Correlation between CFTR gene mutations in Iranian men with congenital absence of the vas deferens and anatomical genital phenotype. J Androl 2008;29:35-40. http://dx.doi.org/10.2164/jandrol.107.002972

Correspondence: Dr Amadou Kassogué, Service d'urologie, CHU Hassan II, Fès, Maroc; amadouenet@yahoo.fr 